\title{
Dipolar correlations in a nanocomposite: A neutron scattering study of Nanoperm $\mathrm{Fe}_{89} \mathrm{Zr}_{7} \mathrm{~B}_{3} \mathrm{Cu}$
}

\author{
A. Michels* \\ Technische Physik, Universität des Saarlandes, Saarbrücken, Germany \\ C. Vecchini and O. Moze \\ CNR-INFM S3 National Research Center, Physics Department, University of Modena and Reggio Emilia, Italy \\ K. Suzuki \\ Department of Materials Engineering, Monash University, Melbourne, Australia \\ P. K. Pranzas \\ GKSS Research Center, Geesthacht, Germany \\ J. Kohlbrecher \\ Paul Scherrer Institute, CH-5232 Villigen PSI, Switzerland \\ J. Weissmüller \\ Institut für Nanotechnologie, Forschungszentrum Karlsruhe, Karlsruhe, Germany \\ and Technische Physik, Universität des Saarlandes, Saarbrücken, Germany
}

(Received 20 June 2006; published 12 October 2006)

\begin{abstract}
We present results for the magnetic-field, temperature, and neutron-polarization dependence of the smallangle neutron scattering intensity in the soft magnetic iron-based nanocomposite Nanoperm $\left(\mathrm{Fe}_{89} \mathrm{Zr}_{7} \mathrm{~B}{ }_{3} \mathrm{Cu}\right)$. $\mathrm{An}$ unusual "clover-leaf-shaped" intensity distribution on the detector is attributed to the dipolar stray fields around the nanosized iron particles, which are embedded in an amorphous magnetic matrix of lesser saturation magnetization. The dipole field induces spin disorder, correlating the spin misalignment of neighboring particles and matrix over several particle spacings. The clover-leaf-shaped anisotropy is observed over a wide range of applied magnetic field and momentum transfer. It persists up to several hundred degrees Kelvin above the Curie temperature of the matrix phase, indicating that some degree of magnetic coupling persists even when the matrix is paramagnetic.
\end{abstract}

DOI: $10.1103 /$ PhysRevB.74.134407

PACS number(s): 75.50.Tt, 61.12.Ex, 75.75.+a

\section{INTRODUCTION}

The interest in nanostructured magnetic materials arises partly from the expectation that novel effects and properties may be expected whenever "crossing length scales" appear in a system, for instance, when the structural building units of a material such as the average particle size, film thickness, or dot size are reduced below a characteristic intrinsic magnetic length scale of the system such as the domain-wall thickness, various magnetic exchange lengths, or the critical single-domain size. ${ }^{1}$ The downscaling of the building blocks of a material to the nanometer scale is accompanied by a continuous demand on observational techniques to resolve ever finer details of the magnetic microstructure.

Magnetic small-angle neutron scattering (SANS) is a method which allows one to analyze the spin structure in the bulk of magnetic materials and on a length scale of about 1-500 nm. As such, the SANS method complements microscopy techniques such as Kerr, Lorentz, ${ }^{2}$ magnetic-force, ${ }^{3}$ (spin-polarized) scanning tunneling microscopy, ${ }^{4,5}$ or photoelectron microscopy, ${ }^{6}$ which are able to image the spin microstructure at surfaces and with a resolution which extends from macroscopic dimensions down to the atomic scale. The range of magnetic materials to which the technique of elastic magnetic SANS has been applied includes, for example, ferrofluids, ${ }^{7-13}$ nanoparticles and precipitates, ${ }^{14-28}$ magnetic recording media, ${ }^{29-32}$ collosal magnetoresistance materials, ${ }^{33-35}$ spin glasses, ${ }^{36-41}$ Invar alloys ${ }^{42,43}$ single crystals, ${ }^{44-46}$ molten elemental ferromagnets, ${ }^{47}$ precipitates in steels, ${ }^{48,49}$ diluted paramagnets in deuterated solutions, ${ }^{50}$ elemental nanocrystalline hard $^{51}$ and soft ${ }^{52-61}$ magnets, as well as hard ${ }^{62,63}$ and soft magnetic ${ }^{64-78}$ nanocomposites. For an early review on the applications of neutron small-angle scattering see Ref. 79 .

A prototypical example for the above-mentioned "crossing-length-scale" scenario are nanocomposites of the Vitroperm (Finemet) and Nanoperm type, which consist of nanoscale Fe-based crystallites embedded in an amorphous magnetic matrix. Herzer's random anisotropy model ${ }^{80}$ is the widely accepted explanation for the extraordinary soft magnetic properties of these materials which result when the average particle size $(D \cong 10-20 \mathrm{~nm})$ is reduced below the magnetic exchange length $(L \cong 35 \mathrm{~nm}){ }^{80}$ By considering the magnetocrystalline and exchange energies, Herzer's model qualitatively rationalizes the dramatic decrease in the coercive field $H_{C}$ at small particle size $\left(H_{C} \propto D^{6}\right)$.

While extensions to the random anisotropy model, including long-range magnetoelastic and induced uniaxial anisotropies, have been brought forward (see, e.g., Ref. 81 and references therein), important interaction terms are notoriously difficult to treat in closed-form theory and are widely neglected. This applies, in particular, to an important term of 
the magnetostatic self-interaction, namely, the nonuniform magnetic field originating from nonzero divergences of the magnetization from within the material, ${ }^{59}$ which act as field sources. The locally nonuniform direction of the magnetization which results from the random anisotropy of the individual nanoparticles is one such source. Even more important can be changes in the magnitude of the magnetization, for instance, free magnetic poles at the phase boundaries between particles and matrix in a composite. Their influence may be expected to be particularly strong during the magnetic-hardening process, ${ }^{82}$ when the decoupling temperature of the intergranular amorphous matrix phase is approached. In the absence of confirmed models for the impact of these effects, it appears particularly important to probe their repercussions by experiments. In fact, a significant impact of internal magnetostatic stray fields on the magnetic microstructure of multiphase nanocrystalline granular systems (and also of nanowires) has previously been demonstrated by means of magnetization measurements, Mössbauer spectroscopy, and electron holography (see, e.g., Refs. 83-89 and references therein).

In two short reports ${ }^{77,78}$ we have recently pointed out that an unusual clover-leaf-shaped ${ }^{90}$ angular anisotropy in the SANS cross section $d \Sigma / d \Omega$ of Nanoperm $\left(\mathrm{Fe}_{89} \mathrm{Zr}_{7} \mathrm{~B}_{3} \mathrm{Cu}\right)$ indicates a decisive influence of the magnetostatic stray field on the spin structure of the material: the jump $\Delta M_{S}$ in the magnitude of the saturation magnetization at the interface between the $\mathrm{Fe}$ particles and the amorphous magnetic matrix gives rise to a dipolar stray field $\mathbf{H}_{D}$ around each crystallite. The component $\mathbf{H}_{D}^{\perp}$ of $\mathbf{H}_{D}$ which is locally perpendicular to the magnetization $\mathbf{M}$ acts as a torque, $\mathbf{H}_{D}^{\perp} \times \mathbf{M}$, so that deflected spins "decorate" the dipole fields, making them visible to the scattering experiment. Thus magnetic neutron scattering-though being sensitive to the Fourier components of the magnetization - can also be used for mapping the distribution of the conjugate quantity, the internal magnetic field.

As an independent confirmation of the findings for Nanoperm, Ogrin et al. ${ }^{32}$ have found evidence for the significance of dipolar stray fields in their SANS study of CoCrPtB-based longitudinal recording media. Figure 1 in their paper $^{32}$ shows a micromagnetic simulation of the spin distribution and of the resulting magnetic SANS intensity, which clearly reveals the disordering effect of the dipole field originating from the cores of the magnetic particles. Likewise, Bischof et al. ${ }^{91}$ have directly observed a cloverleaf-shaped anisotropy in the field-dependent SANS cross section resulting from nanometer-sized $\mathrm{NiAl}$ precipitates in steels.

Here, we report the results of temperature and neutronpolarization-dependent SANS experiments on Nanoperm, with particular attention to the effect of the magnetic ordering transition of the amorphous matrix at $T_{C}^{\mathrm{am}}=345 \mathrm{~K}$ on the dipolar interaction and on the magnetic correlations between the Fe nanoparticles. We derive an expression for the polarization-dependent nuclear and magnetic small-angle neutron scattering cross section which contains anisotropic contributions that explain our experimental observations. The paper is organized as follows: In Sec. II we describe the SANS experiments and the sample characterization. In Secs.
III A and III B we provide an analysis of the elastic magnetic small-angle neutron scattering cross section of a multicomponent and multiphase nanocrystalline ferromagnet. In Secs. IV A-IV C we present, respectively, the results of the magnetic-field, temperature, and neutron-polarization dependence of the dipole-field-induced spin-misalignment scattering, and in Sec. IV D we show the results of magnetization measurements. Secs. V A-V D provide a discussion of the experimental data. Finally, Sec. VI summarizes the main results of this study.

\section{EXPERIMENTAL PROCEDURE}

The SANS experiments were performed at the SANS-1 instrument $^{92}$ at the Paul Scherrer Institute (PSI), Villigen, Switzerland, and at the SANS-2 instrument ${ }^{93}$ at the Geesthacht Neutron Facility (GeNF), Geesthacht, Germany. We used incident neutron wavelengths of $\lambda=6.0 \AA$ (PSI) and $\lambda$ $=5.8 \AA(\mathrm{GeNF})$ with a respective wavelength spread $\Delta \lambda / \lambda$ $=10 \%$ full width at half maximum. At PSI, a remanent supermirror transmission polarizer, mounted in the first collimator drum behind the neutron velocity selector, and a rf spin flipper near the sample position, allowed one to select and to reverse the polarization state of the incident neutrons. The applied magnetic field $H$ was provided by an electromagnet with the field direction horizontal and in the plane of the ribbon sample. The measurements at GeNF served to establish the field-dependent scattering cross section $d \Sigma / d \Omega$ at room temperature. Supplementary runs were carried out at PSI to investigate the variation of the scattering with temperature (in the interval $T=298-693 \mathrm{~K}$ ) and with the neutron-polarization state. In the interest of exploring a maximum range of $H$ and $T$ within the limited beamtime, and consistent with the emphasis on variations of the scattering, no instrumental background was recorded for this part of the data. During the temperature scans the sample was held in a vacuum of $1.0 \times 10^{-4}$ mbar. Temperature stability was $\pm 2 \mathrm{~K}$. The SANS data reduction was carried out using the BerSANS $^{94}$ (PSI) and SANDRA ${ }^{93}(\mathrm{GeNF})$ software packages.

The Nanoperm $\left(\mathrm{Fe}_{89} \mathrm{Zr}_{7} \mathrm{~B}_{3} \mathrm{Cu}\right)$ sample was prepared by melt spinning, followed by a subsequent annealing treatment for $1 \mathrm{~h}$ at $745 \mathrm{~K}$, which resulted in the precipitation of bcc Fe particles in an amorphous magnetic matrix. The average Fe particle size $D=12 \mathrm{~nm}$ was determined by x-ray diffraction and transmission electron microscopy. From Mössbauer experiments, ${ }^{74}$ similar to the ones reported in Ref. 82, we have estimated the Curie temperature of the amorphous matrix phase at $T_{C}^{\mathrm{am}} \cong 345 \mathrm{~K}$ and the crystalline volume fraction $x_{C} \cong 40 \%$. For the SANS measurements several ribbons of typical dimensions $20 \times 2 \times 0.02 \mathrm{~mm}$ were stacked and mounted on a $\mathrm{Cd}$ aperture. Sample transmission varied between $96.7 \%$ at $\mu_{0} H=1.5 \mathrm{~T}$ and $94.5 \%$ at $30 \mathrm{mT}$, so that multiple-scattering effects are expected to be negligible. ${ }^{95}$

After the exposition to a temperature of $693 \mathrm{~K}$ for several hours, the SANS sample retained its shiny metallic surface. Subsequent investigations with transmission electron microscopy and $\mathrm{x}$-ray diffraction revealed that the average particle size remained unaffected by the heat treatment. Furthermore, temperature scans and an isothermal measurement with dif- 
ferential scanning calorimetry at $T=695 \mathrm{~K}$ for several hours confirmed the absence of significant changes in the microstructure of the material.

Magnetization measurements were performed with a commercial superconducting quantum interference device magnetometer (Quantum Design MPMS-7) up to applied magnetic fields of $\mu_{0} H=7 \mathrm{~T}$ and in the temperature range from 5 to $300 \mathrm{~K}$.

\section{THE SMALL-ANGLE NEUTRON SCATTERING CROSS SECTION OF A MULTICOMPONENT AND MULTIPHASE NANOCRYSTALLINE FERROMAGNET}

\section{A. Discrete array of atoms}

We base our discussion of magnetic small-angle neutron scattering (SANS) on the expressions for the spin-dependent partial cross sections given by Moon, Riste, and Koehler (MRK). ${ }^{96}$ Detailed accounts of magnetic neutron scattering can also be found in the treatises by Lovesey, Williams, and Hicks. ${ }^{97-99}$ The MRK formulas have already been discussed previously with relation to field-dependent SANS; in each case, simplified versions were employed, ignoring terms that are of no consequence for single-phase materials. ${ }^{51,56,100} \mathrm{Ex}$ periments on nanocomposites require more general formulas, allowing for nonuniform values of density, composition, and magnitude of the magnetization, and for a systematic correlation of these parameters to the orientation of the atomic magnetic moments. Based on our experimental observations, it is not possible to use approaches, such as the one of Ref. 61 , which treat the composite as an array of uniformly magnetized homogeneous particles in a nonmagnetic or partially polarized homogeneous matrix: the metallic nanocomposites of interest afford no a priori justification for neglecting the contribution of nanoscale structure in the spin system of matrix and/or crystallites to the scattering. Here, we apply the MRK equations to a suitably generalized case.

The elastic differential scattering cross section $d \Sigma / d \Omega$ at scattering vector $\mathbf{q}$ due to an arrangement of atoms at positions $\mathbf{x}_{i}$ can be written as ${ }^{96}$

$$
\begin{aligned}
\frac{d \Sigma^{ \pm \pm}}{d \Omega}(\mathbf{q})= & \frac{1}{V} \sum_{i, j} \exp \left(-i \mathbf{q} \mathbf{x}_{i, j}\right) \\
& \times\left[b_{n, i} b_{n, j}^{*} \pm b_{n, i} b_{m, j}^{*} Q_{z, j}^{*} \pm b_{n, j}^{*} b_{m, i} Q_{z, i}\right. \\
& \left.+b_{m, i} b_{m, j}^{*} Q_{z, i} Q_{z, j}^{*}\right], \\
\frac{d \Sigma^{ \pm \mp}}{d \Omega}(\mathbf{q})= & \frac{1}{V} \sum_{i, j} \exp \left(-i \mathbf{q} \mathbf{x}_{i, j}\right) b_{m, i} b_{m, j}^{*} \\
& \times\left[Q_{x, i} Q_{x, j}^{*}+Q_{y, i} Q_{y, j}^{*} \mp i \mathbf{e}_{z} \cdot\left(\mathbf{Q}_{i} \times \mathbf{Q}_{j}^{*}\right)\right] .
\end{aligned}
$$

In Eqs. (1) and (2) we have neglected nuclear spin-dependent scattering; the quantity $V$ denotes the scattering volume, $\mathbf{x}_{i, j}=\mathbf{x}_{i}-\mathbf{x}_{j}$, the parameters $b_{n, i}$ and $b_{m, i}$ represent, respectively, the atomic coherent nuclear and magnetic scattering lengths of atom " $i$," the superscript asterisk refers to the complex conjugated quantity, and the Halpern-Johnson vector $\mathbf{Q}_{i}=\boldsymbol{\epsilon}\left(\boldsymbol{\epsilon} \cdot \mathbf{m}_{i}\right)-\mathbf{m}_{i}($ Ref. 101) is related to the unit vector $\boldsymbol{\epsilon}$ along the direction of $\mathbf{q}$ and to the unit vector $\mathbf{m}_{i}$ $=\left(m_{x, i}, m_{y, i}, m_{z, i}\right)$ along the magnetization. The superscripts to $d \Sigma / d \Omega$ relate to the spin state of the incident and of the scattered neutron, relative to the polarization axis of the incident neutron beam, which is parallel to the applied magnetic field $\mathbf{H}=H \mathbf{e}_{z}$. The four partial cross sections $d \Sigma^{ \pm \pm} / d \Omega$ and $d \Sigma^{ \pm \mp} / d \Omega$ denote, respectively, the "non-spin-flip" and "spin-flip" scattering cross sections, where the spin of the incident and scattered neutron is, respectively, in the " $+(-)$ " and "+(-)" and in the "+(-)" and " $-(+)$ " direction. ${ }^{96}$

In general, SANS instrumentation does not allow for polarization analysis. Therefore, only combinations of the partial cross sections can be measured. These measurable quantities, denoted by $d \Sigma^{+} / d \Omega$ and $d \Sigma^{-} / d \Omega$, combine non-spinflip and spin-flip scattering contributions. In particular, $d \Sigma^{+} / d \Omega=d \Sigma^{++} / d \Omega+d \Sigma^{+-} / d \Omega$ and $\quad d \Sigma^{-} / d \Omega=d \Sigma^{--} / d \Omega$ $+d \Sigma^{-+} / d \Omega$.

Taking $\mathbf{H}$ along $\mathbf{e}_{z}$ and the incident neutron beam along $\mathbf{e}_{x}$, we can write $\boldsymbol{\epsilon}=\mathbf{q} / q \cong(0, \sin \theta, \cos \theta)$, where $\theta$ denotes the angle included between $\mathbf{q}$ and $\mathbf{H}$. Hence

$$
\mathbf{Q}=\left(\begin{array}{c}
-m_{x} \\
-m_{y} \cos ^{2} \theta+m_{z} \sin \theta \cos \theta \\
-m_{z} \sin ^{2} \theta+m_{y} \sin \theta \cos \theta
\end{array}\right) .
$$

It is generally desirable to insert the above expression for $\mathbf{Q}_{i}$ into Eqs. (1) and (2), and to collect the terms containing the transverse magnetization components $m_{x}$ and $m_{y}$ in a separate term, the so-called spin-misalignment scattering cross section $d \Sigma_{M}^{ \pm} / d \Omega$, which vanishes at saturation when all the magnetic moments are aligned along the applied-field direction. The total $d \Sigma^{ \pm} / d \Omega$ can then be written as the sum of $d \Sigma_{M}^{ \pm} / d \Omega$ and of the residual scattering cross section $d \Sigma_{R}^{ \pm} / d \Omega$, which contains all the remaining terms, ${ }^{56}$

$$
\begin{aligned}
& \frac{d \Sigma^{ \pm}}{d \Omega}(\mathbf{q})=\frac{d \Sigma_{R}^{ \pm}}{d \Omega}(\mathbf{q})+\frac{d \Sigma_{M}^{ \pm}}{d \Omega}(\mathbf{q}), \\
\frac{d \Sigma_{R}^{ \pm}}{d \Omega}(\mathbf{q})= & \frac{1}{V} \sum_{i, j} \exp \left(-i \mathbf{q} \mathbf{x}_{i, j}\right) \\
\times & {\left[b_{n, i} b_{n, j}^{*} \mp\left(b_{n, i} b_{m, j}^{*} m_{z, j}+b_{n, j}^{*} b_{m, i} m_{z, i}\right) \sin ^{2} \theta\right.} \\
+ & \left.b_{m, i} b_{m, j}^{*} m_{z, i} m_{z, j} \sin ^{2} \theta\right], \\
\frac{d \Sigma_{M}^{ \pm}}{d \Omega}(\mathbf{q})= & \frac{1}{V} \sum_{i, j} \exp \left(-i \mathbf{q} \mathbf{x}_{i, j}\right) \\
& \times\left[ \pm\left(b_{n, i} b_{m, j}^{*} m_{y, j}+b_{n, j}^{*} b_{m, i} m_{y, i}\right) \sin \theta \cos \theta\right. \\
& +b_{m, i} b_{m, j}^{*}\left(m_{x, i} m_{x, j}+m_{y, i} m_{y, j} \cos ^{2} \theta\right. \\
& \left.\left.-\left(m_{y, i} m_{z, j}+m_{y, j} m_{z, i}\right) \sin \theta \cos \theta\right)\right] .
\end{aligned}
$$

We have neglected in Eqs. (5) and (6) the contributions due to $\mathbf{Q}_{i} \times \mathbf{Q}_{j}$ [compare Eq. (2)]. These terms depend on the angle included by the pair of spins $i$ and $j$, and, unless the spins take a helical structure with a preferred direction of rotation, they will take on either sign with equal probability. Therefore for simple ferromagnets or antiferromagnets, the 
cross-product terms do not generally contribute to the sum in Eq. (2). ${ }^{102}$

It is seen that the angular anisotropy of the residual scattering cross section follows the familiar $d \Sigma_{R} / d \Omega \propto \sin ^{2} \theta$ behavior, resulting in an enhanced magnetic intensity perpendicular to the net magnetization, as is characteristic, for instance, for saturated magnetic particles in a nonmagnetic matrix.

The explicit angular dependency of the spin-misalignment contribution is distinctly different from that of the residual scattering; it contains terms $d \Sigma_{M} / d \Omega \propto \sin \theta \cos \theta$ and $d \Sigma_{M} / d \Omega \propto \cos ^{2} \theta$. Note that correlations between the spin components along the incident beam, $m_{x}$, give rise to an isotropic scattering contribution to $d \Sigma_{M} / d \Omega$. As a result of interference between nuclear and magnetic scattering, both $d \Sigma_{R} / d \Omega$ and $d \Sigma_{M} / d \Omega$ depend on the polarization of the incident neutron beam. We also would like to emphasize that the terms in $d \Sigma_{M} / d \Omega$ which are proportional to $\cos ^{2} \theta$ depend on the square of the transversal spin component $m_{y}$, while terms proportional to $\sin \theta \cos \theta$ depend only linearly on $m_{y}$.

\section{B. Continuum formulation}

Since the discreteness of the atomic structure of matter is generally of no importance in small-angle scattering, the scattering cross section can equivalently be expressed in terms of continuous Fourier transforms of suitably coarsegrained, continuous distributions of nuclear and magnetic scattering-length densities, $N(\mathbf{x})$ and $\mathbf{M}(\mathbf{x})$, respectively. Generally, it is convenient to work with these continuous quantities, since models used in analyzing the results of SANS experiments are most often also based on a continuum picture, embodying geometric concepts such as spheres or rods, alloy concentration profiles, or the continuous magnetization profiles of micromagnetics.

We define the continuous nuclear, $N(\mathbf{x})$, and magnetic, $\mathbf{M}(\mathbf{x})$, scattering-length densities along with their respective Fourier transforms, $\tilde{N}(\mathbf{q})$ and $\tilde{\mathbf{M}}(\mathbf{q})$, via

$$
\begin{gathered}
N(\mathbf{x})=\sum_{\alpha} b_{n, \alpha} \rho_{\alpha}(\mathbf{x}), \\
\mathbf{M}(\mathbf{x})=\sum_{\alpha} \mu_{\alpha} \rho_{\alpha}(\mathbf{x}) \mathbf{m}_{\alpha}(\mathbf{x}), \\
\tilde{N}(\mathbf{q})=\frac{1}{(2 \pi)^{3 / 2}} \int N(\mathbf{x}) \exp (-i \mathbf{q} \mathbf{x}) d^{3} x, \\
\tilde{\mathbf{M}}(\mathbf{q})=\frac{1}{(2 \pi)^{3 / 2}} \int \mathbf{M}(\mathbf{x}) \exp (-i \mathbf{q} \mathbf{x}) d^{3} x .
\end{gathered}
$$

Here, the index $\alpha$ labels the alloy component, $\rho_{\alpha}(\mathbf{x})$ denotes the atomic density, $\mu_{\alpha}$ denotes the magnitude of the atomic magnetic moment, and $\mathbf{m}_{\alpha}$ is a unit vector along the locally averaged orientation of the moments of component $\alpha$. Using Eqs. (7)-(10), $d \Sigma_{R} / d \Omega$ and $d \Sigma_{M} / d \Omega$ can be expressed as

$$
\begin{gathered}
\frac{d \Sigma_{R}^{ \pm}}{d \Omega}(\mathbf{q})=\frac{8 \pi^{3}}{V}\left[|\tilde{N}|^{2} \mp b_{H}\left(\tilde{N} \tilde{M}_{z}^{*}+\tilde{N}^{*} \tilde{M}_{z}\right) \sin ^{2} \theta\right. \\
\left.+b_{H}^{2}\left|\tilde{M}_{z}\right|^{2} \sin ^{2} \theta\right] \\
\frac{d \Sigma_{M}^{ \pm}}{d \Omega}(\mathbf{q})=\frac{8 \pi^{3}}{V}\left[ \pm b_{H}\left(\tilde{N} \tilde{M}_{y}^{*}+\tilde{N}^{*} \tilde{M}_{y}\right) \sin \theta \cos \theta+b_{H}^{2}\left|\tilde{M}_{x}\right|^{2}\right. \\
\left.+b_{H}^{2}\left|\tilde{M}_{y}\right|^{2} \cos ^{2} \theta-b_{H}^{2}\left(\tilde{M}_{y} \tilde{M}_{z}^{*}+\tilde{M}_{y}^{*} \tilde{M}_{z}\right) \sin \theta \cos \theta\right]
\end{gathered}
$$

where $b_{H}=2.7 \times 10^{-15} \mathrm{~m} / \mu_{B},{ }^{100,103}$ and the subscripts $x, y$, and $z$ refer to the Cartesian components of $\tilde{\mathbf{M}}$. The magnetic form factor $f$ in the expression for the magnetic scattering length $b_{m}=2.7 \times 10^{-15} \mathrm{~m} f \mu / \mu_{B}{ }^{96,101}$ was set to unity, which is permissible in the small-angle region. Since SANS is sensitive to deviations of the local scattering-length density from its (constant) mean value, Eqs. (11) and (12) remain valid at $\mathbf{q} \neq 0$ if $\tilde{N}$ and $\tilde{\mathbf{M}}$ are replaced by the corresponding excess scattering-length densities $\Delta \tilde{N}$ and $\Delta \tilde{\mathbf{M}}$, which are obtained by subtracting arbitrary constants from the realspace variables, for instance, the respective macroscopic averages.

Equations (11) and (12) account quite generally for the combined nuclear and magnetic small-angle scattering from a multicomponent and multiphase system, except for the neglect of helical spin structures (e.g., Ref. 46) and nuclear spin-dependent scattering (e.g., Ref. 50). As compared to earlier studies of magnetic SANS, ${ }^{51,56}$ which used simpler equations matched to single-component systems, the present results contain extra terms accounting for interference between scattering due to the transverse magnetization components and due to variations of the alloy composition. This latter quantity affects the nuclear scattering-length density as well as the magnitude of the magnetization and, thereby, $M_{z}$. The extra terms exhibit a $\sin \theta \cos \theta$ angular variation of $d \Sigma_{M} / d \Omega$, which is not found in the simpler formula, ${ }^{56}$ and they have a linear rather than a quadratic dependence on $M_{y}$. For the particular alloy samples considered in the present experiments, it turns out that this scattering contribution is actually the dominant one at intermediate applied magnetic fields. An important consequence is that the equations of Ref. 56 , which relate the $q$ - and $\theta$-dependence of the terms in $d \Sigma_{M} / d \Omega$ (which are quadratic in $M_{x}$ and $M_{y}$ ) to the magnetic interaction terms, do not provide the complete picture here.

In the approach-to-saturation regime, $d \Sigma_{R} / d \Omega$ is approximately independent of the applied magnetic field, whereas $d \Sigma_{M} / d \Omega$ exhibits an extraordinary large dependence on $H$ (compare, e.g., Figs. 5 and 10 in Ref. 56). When the sample is saturated, $d \Sigma_{M} / d \Omega$ is zero, and subtraction of $d \Sigma / d \Omega$ $=d \Sigma_{R} / d \Omega$ from a measurement of $d \Sigma / d \Omega$ at lower field yields the spin-misalignment scattering cross section at that particular field value. In the following analysis of the experimental data we consider the scattering at the highest applied magnetic field as an approximation to the scattering at saturation, and we shall discuss the corresponding differenceintensity data with relation to the spin-misalignment scattering. 


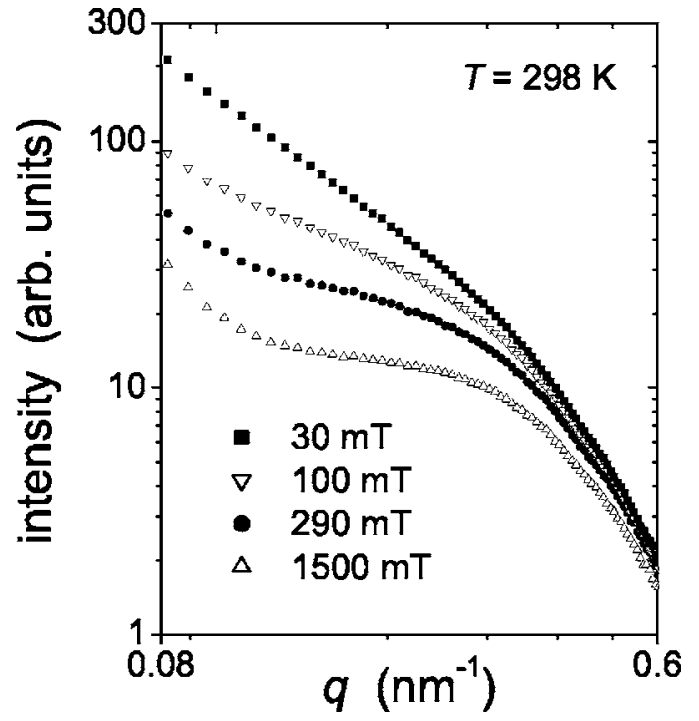

FIG. 1. Log-log plot of the radially averaged total SANS intensity of Nanoperm $\left(\mathrm{Fe}_{89} \mathrm{Zr}_{7} \mathrm{~B}_{3} \mathrm{Cu}\right)$ at room temperature as a function of the applied magnetic field (see inset). Data is not corrected for background scattering.

As has been shown previously ${ }^{51,56}$ for typical values of the spin-wave stiffness constant and spin-wave gap, inelastic (magnon) scattering contributions are suppressed throughout the major part of the parameter space explored in SANS experiments such as the one reported here. Therefore the SANS signal is entirely elastic.

\section{EXPERIMENTAL RESULTS}

\section{A. Field dependence of SANS}

Figure 1 depicts the radially averaged total (nuclear and magnetic) SANS intensity of Nanoperm at room temperature as a function of the external magnetic field. Note that, unlike the difference-intensity data [Figs. 3-6, 7(a), 8, and 9 below], the data shown in Fig. 1 is not corrected for instrumental background scattering. This does, however, not influence the following qualitative discussion of the field dependence of the scattering.

It is seen that between $\mu_{0} H=30$ and $1500 \mathrm{mT}$ - a field regime where the sample is in the approach-to-saturation regime (compare Fig. 10 below) - the scattering exhibits a strong field dependence; the SANS signal at the smallest $q$-values decreases by almost one order of magnitude.

In assessing the data of Fig. 1 it is important to note that the sample is in a single-domain state at all fields shown. This follows from the estimated value of the volumetric demagnetizing field ${ }^{104}$ of the SANS specimen, about $20 \mathrm{mT},{ }^{77}$ and from the expectation that, as a consequence of the extreme softness of Nanoperm, the SANS sample is a single domain for applied fields larger than its demagnetizing field. Thus the data in Fig. 1 demonstrate that the origin of the scattering is unrelated to a macroscopic domain structure. Instead, the scattering arises from nanoscale spinmisalignment, i.e., from the failure of the spins to completely align with the external field.
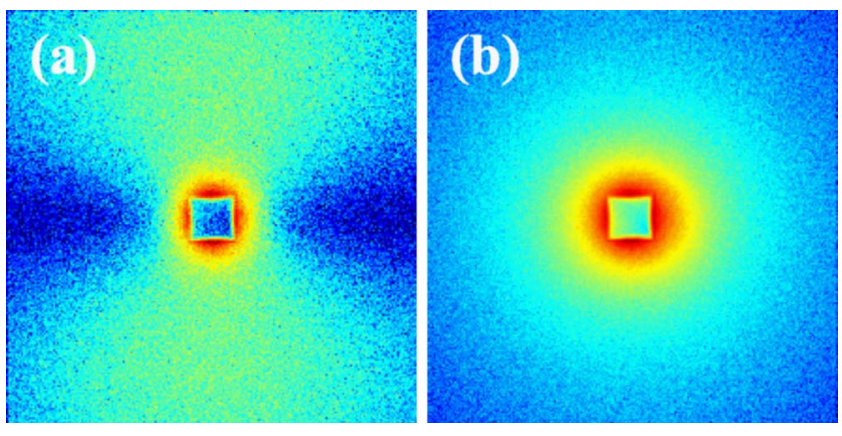

FIG. 2. (Color online) Room temperature total SANS cross sections $d \Sigma / d \Omega$ of Nanoperm $\left(\mathrm{Fe}_{89} \mathrm{Zr}_{7} \mathrm{~B}_{3} \mathrm{Cu}\right)$ (logarithmic scale). The applied magnetic field $\mathbf{H}$ is horizontal, as in Fig. 3. (a) $\mu_{0} H$ $=1994 \mathrm{mT}$ and (b) $0 \mathrm{mT}$. Pixels in the corners have momentum transfer $q=0.48 \mathrm{~nm}^{-1}$.

At $\mu_{0} H=2 \mathrm{~T}$, when the sample is essentially saturated, the intensity distribution on the two-dimensional detector exhibits the well-known $\sin ^{2} \theta$ anisotropy with maximum intensity perpendicular to $\mathbf{H}$, indicating a saturated magnetic microstructure [compare Fig. 2(a)]. By contrast, at zero field [Fig. 2(b)], $d \Sigma / d \Omega$ is isotropically distributed, which suggests a random orientation of domains and the absence of significant induced uniaxial anisotropy.

Figure 3 shows difference-intensity data at several applied magnetic fields where the total nuclear and magnetic SANS intensity at $\mu_{0} H=1.5 \mathrm{~T}$ has been subtracted from the respective measurement.

In a range of intermediate field values (30-290 mT), the difference images reveal a pronounced angular anisotropy with maxima at angles $\theta \cong \pm 30^{\circ}-40^{\circ}$ relative to $\mathbf{H}$. This clover-leaf-shaped anisotropy is already visible in the raw data, and the subtraction procedure merely serves to make it all the more evident. The feature is absent close to saturation and at zero field (compare Fig. 2). Field-dependent SANS measurements with a large sample-to-detector distance of $20 \mathrm{~m}$ gave no indication of the presence of the clover-leafshaped angular anisotropy at the smallest scattering angles $\left(q \cong 0.01 \mathrm{~nm}^{-1}\right)$. Instead, the SANS data at the smallest $q$-values revealed the usual $\left(1+\cos ^{2} \theta\right)$ type intensity variation, in agreement with the prediction for long-range spinmisalignment scattering. ${ }^{56}$
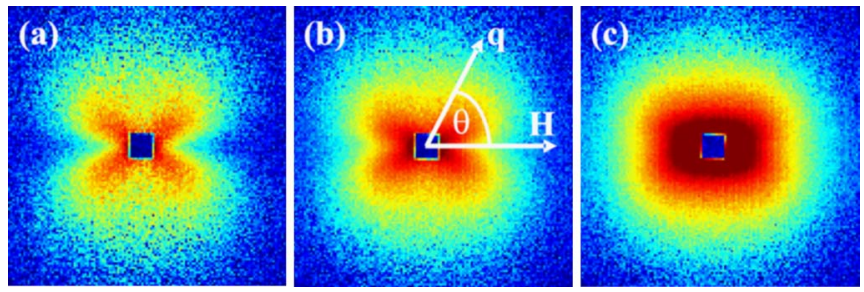

FIG. 3. (Color online) Field dependence of difference-intensity data of Nanoperm $\left(\mathrm{Fe}_{89} \mathrm{Zr}_{7} \mathrm{~B}_{3} \mathrm{Cu}\right)$ at $T=298 \mathrm{~K}$ (logarithmic scale). The total SANS intensity at $\mu_{0} H=1.5 \mathrm{~T}$ has been subtracted from the respective SANS signal at the lower field. $\theta$ is the angle between the scattering vector $\mathbf{q}$ and the applied magnetic field $\mathbf{H}$. Pixels in the corners have momentum transfer $q=0.64 \mathrm{~nm}^{-1}$. Values of the applied field (in mT): (a) 290, (b) 100, and (c) 30 . 

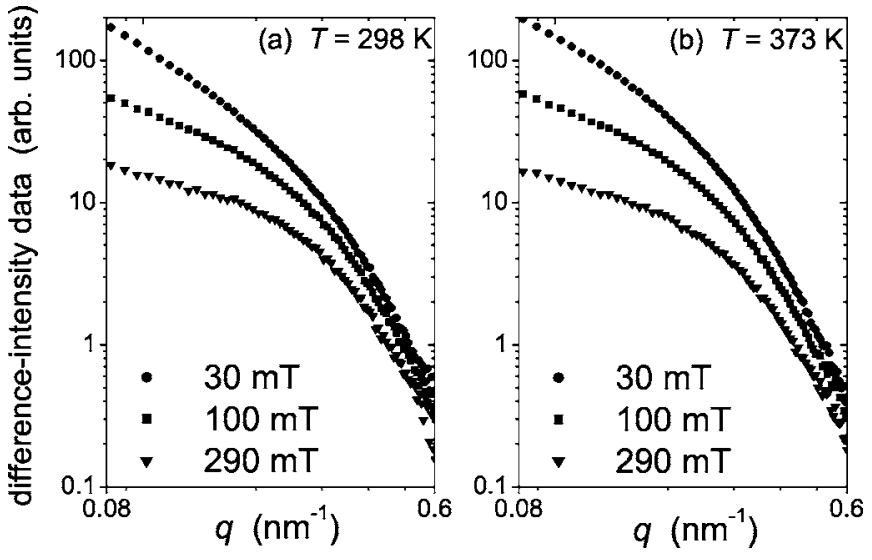

FIG. 4. Log-log plot of the radially averaged difference SANS intensities of Nanoperm $\left(\mathrm{Fe}_{89} \mathrm{Zr}_{7} \mathrm{~B}_{3} \mathrm{Cu}\right)$ as a function of the applied magnetic field (see inset). (a) $T=298 \mathrm{~K}$ and (b) $T=373 \mathrm{~K}$.

With decreasing applied field, the clover-leaf-shaped pattern in Fig. 3 is progressively "washed out," and the overall magnetic SANS intensity increases. Figure 4 shows the field dependence of the radially averaged difference-intensity data at two temperatures, 298 and $373 \mathrm{~K}$.

At both temperatures, increasing the applied field from 30 to $290 \mathrm{mT}$ leads to a suppression of the amplitude of the transversal spin fluctuations, as can be inferred from the observation that the spin-misalignment scattering decreases (compare also Fig. 1). The relatively strong field dependence of the difference-intensity signal prevails over a wide range in temperature and momentum transfer, between about $0.08 \mathrm{~nm}^{-1} \leqslant q \leqslant 0.6 \mathrm{~nm}^{-1}(10 \mathrm{~nm} \leqslant 2 \pi / q \leqslant 80 \mathrm{~nm})$.

\section{B. Temperature dependence of SANS}

Figure 5 shows the temperature dependence of the difference-intensity detector pattern at a fixed external field of $\mu_{0} H=290 \mathrm{mT}$. The clover-leaf-shaped anisotropy remains clearly visible for temperatures up to $T=693 \mathrm{~K}$, i.e., far above the Curie temperature $T_{C}^{\mathrm{am}}=345 \mathrm{~K}$ of the amorphous matrix phase, which was determined from Mössbauer measurements. ${ }^{74}$

Figure 6 represents a log-log plot of the temperature variation of the radially averaged difference SANS data at $290 \mathrm{mT}$. With increasing temperature the radially averaged data first take on a maximum at $323 \mathrm{~K}$ [not shown in Fig. 6, see also Fig. 7(a)], and then, on increasing the temperature further up to $693 \mathrm{~K}$, the difference intensity decreases by a factor of about 2 at the smallest momentum transfers.

The temperature dependence of the corresponding integrated difference-intensity data at $290 \mathrm{mT}$ and of the integrated total nuclear and magnetic SANS intensity at $1500 \mathrm{mT}$ can be seen in Fig. 7(a).

Close to saturation, at $\mu_{0} H=1.5 \mathrm{~T}$, where the scattering originates dominantly from the spatial variation of the magnitude of the magnetization, the integrated total intensity increases up to $T=373 \mathrm{~K}$, and it then enters a plateau region with no significant change up to $693 \mathrm{~K}$, in agreement with the results of Ref. 74. This is well compatible with the expected temperature variation of $\Delta M_{S}$ which is shown sche-
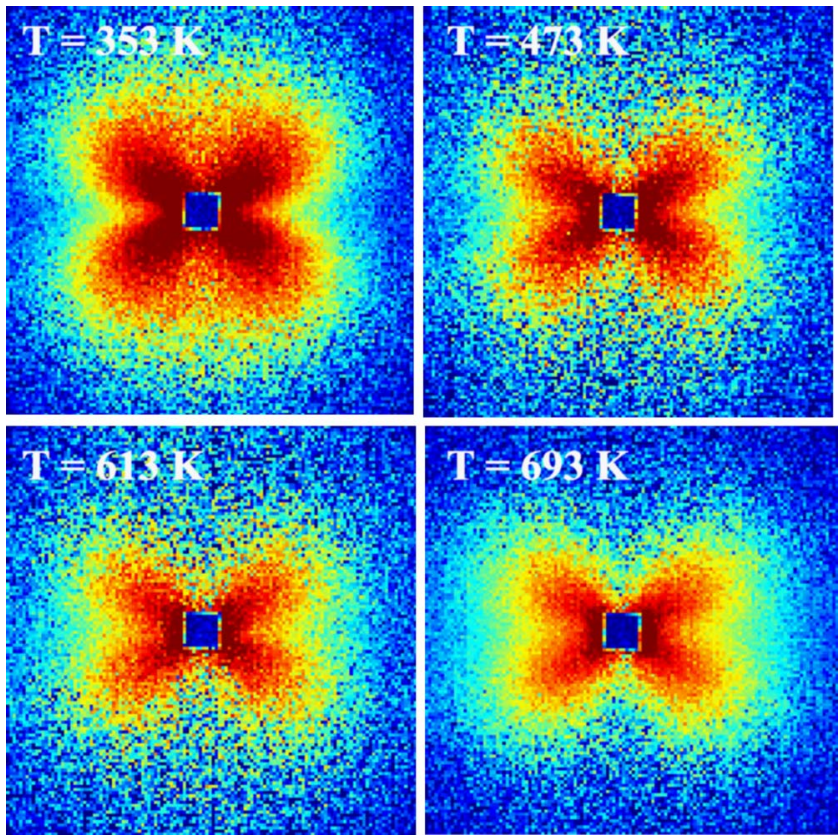

FIG. 5. (Color online) Temperature variation of differenceintensity data of Nanoperm $\left(\mathrm{Fe}_{89} \mathrm{Zr}_{7} \mathrm{~B}_{3} \mathrm{Cu}\right)$ at $\mu_{0} \mathrm{H}=290 \mathrm{mT}$ (logarithmic scale). The external field $\mathbf{H}$ is applied horizontally. The total SANS intensity at $\mu_{0} H=1.5 \mathrm{~T}$ and at the respective temperature has been subtracted from the respective SANS signal at $290 \mathrm{mT}$. Pixels in the corners have momentum transfer $q=0.64 \mathrm{~nm}^{-1}$.

matically in Fig. 7(b): a comparatively pronounced increase near $T_{C}^{\mathrm{am}}$ where $M_{S}$ of the matrix vanishes, and a weak decrease at higher $T$, reflecting the temperature-dependent $M_{S}$ of the crystallites. Similar observations on a Finemet-type alloy were made by Kohlbrecher et al. ${ }^{67}$ The analogous data for the spin-misalignment scattering at $290 \mathrm{mT}$ exhibits a shallow peak at $323 \mathrm{~K}$ followed by a slight decrease at higher $T$.

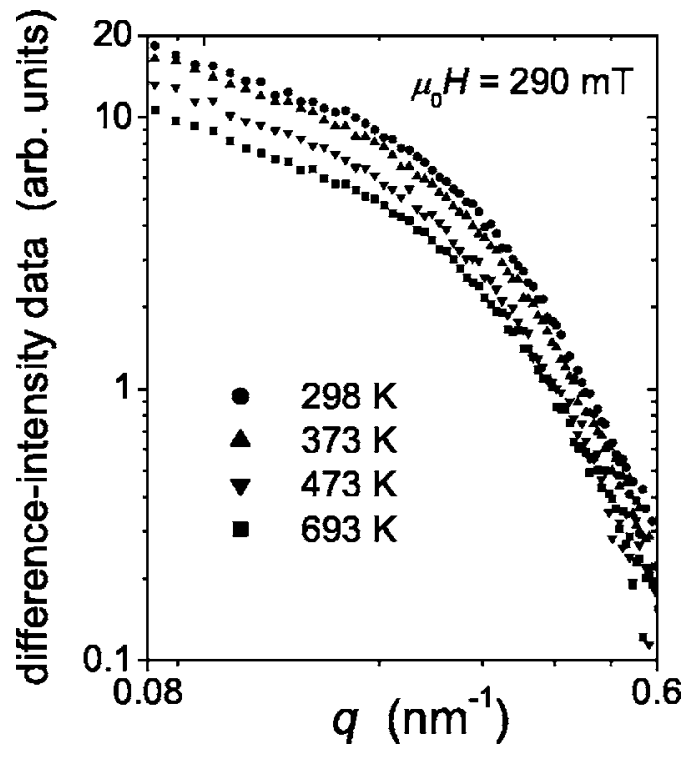

FIG. 6. Radially averaged difference-intensity data of Nanoperm $\left(\mathrm{Fe}_{89} \mathrm{Zr}_{7} \mathrm{~B}_{3} \mathrm{Cu}\right.$ ) at $\mu_{0} \mathrm{H}=290 \mathrm{mT}$ as a function of temperature (see inset). 

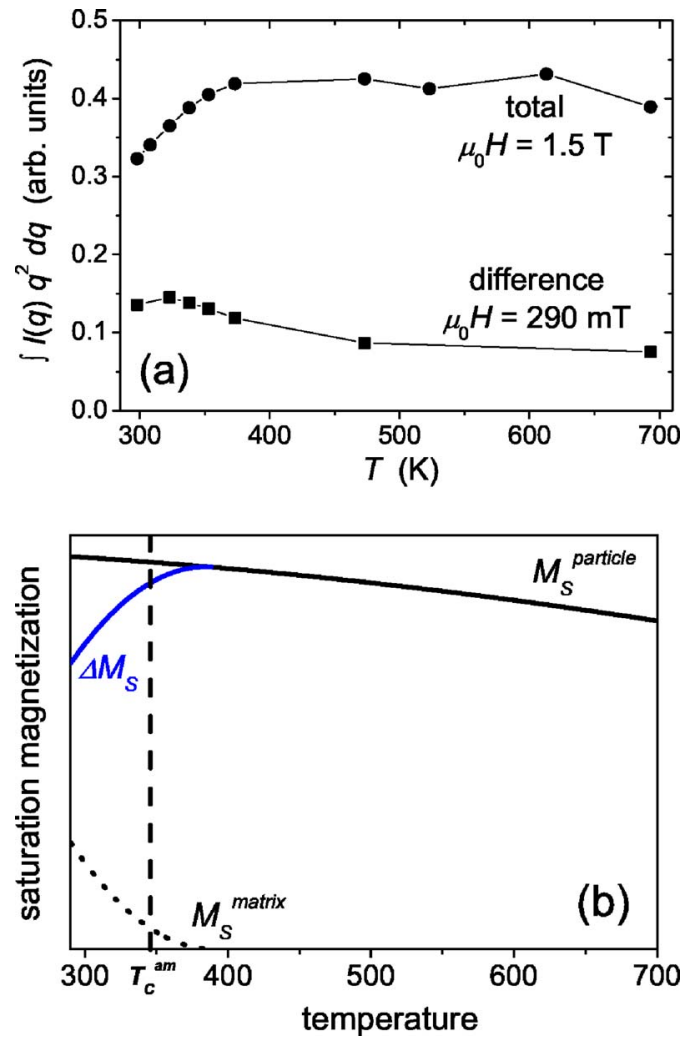

FIG. 7. (a) (Color online) Temperature dependence of the integrated total SANS intensity at $\mu_{0} H=1500 \mathrm{mT}$ and of the integrated difference intensity at $\mu_{0} H=290 \mathrm{mT}$. The respective integrated intensity was calculated according to $\int_{q_{\min }}^{q_{\max }} I(q) q^{2} d q$ with $q_{\min }$ $=0.08 \mathrm{~nm}^{-1}$ and $q_{\max }=0.6 \mathrm{~nm}^{-1}$. (b) Qualitative temperature dependences of the saturation magnetizations $M_{S}$ of the Fe particles and of the amorphous magnetic matrix. The difference $\Delta M_{S}$ between both $M_{S}(T)$ curves is related to the magnetic SANS contrast which is due to spatial variations $M_{S}(\mathbf{x})$ of the magnitude of the magnetization.

\section{Neutron-polarization dependence of SANS}

Figure 8 depicts a plot of the difference between the SANS intensities with the neutron-spin flipper on, $I^{-}$, and off, $\mathrm{I}^{+}$, at selected values of the external field. At all fields does the intensity distribution clearly reveal a dominating $\sin ^{2} \theta$ behavior with an enhanced SANS signal normal to the field direction. The clover-leaf-shaped angular anisotropy, which is visible in the unpolarized data (Fig. 3), is absent. This observation is remarkable in view of Eqs. (5) and (6) for the residual and spin-misalignment scattering cross section, which contain terms $\mp b_{n} b_{m} m_{z} \sin ^{2} \theta$ and, in particular, $\pm b_{n} b_{m} m_{y} \sin \theta \cos \theta$ that do depend on the polarization of the incident neutron beam. The radially averaged "flipper onflipper off" data is shown in Fig. 9.

Compared to the data taken with an unpolarized incident beam (Fig. 4), the difference between the two spin states, $I^{-}-I^{+}$, is small in magnitude, and it is only weakly dependent on the applied magnetic field.

\section{Magnetization data}

In Fig. 10 we plot the room temperature magnetization isotherm and in Fig. 11 the applied-field dependence of the
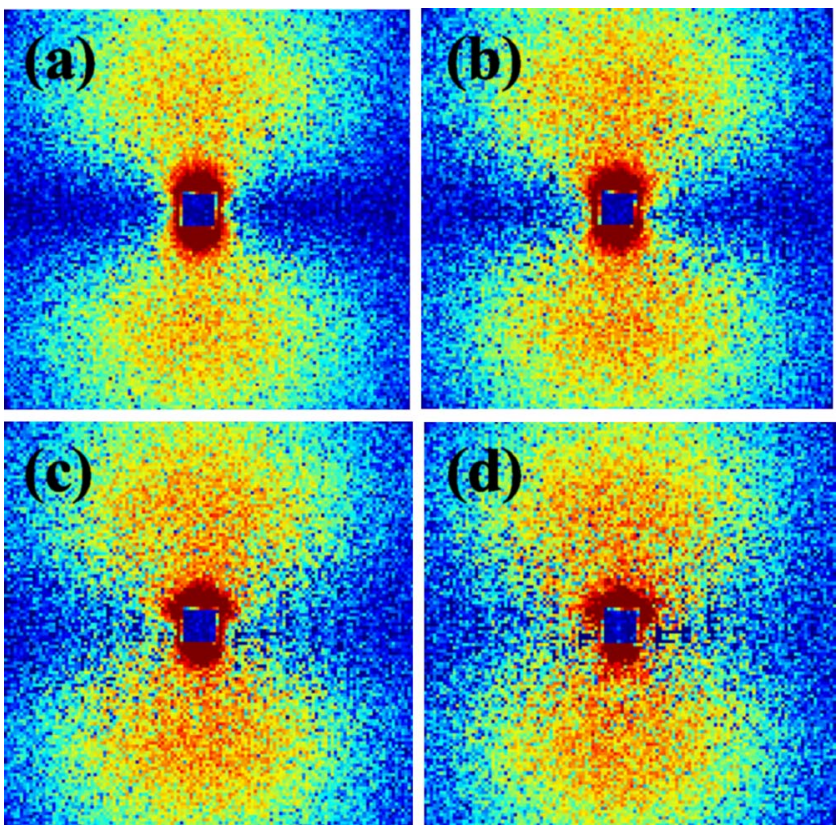

FIG. 8. (Color online) Difference between flipper on and flipper off SANS intensities, $I^{-}-I^{+}$, as a function of the applied magnetic field (logarithmic scale). $\mathbf{H}$ is horizontal. Data were recorded at room temperature. Pixels in the corners have momentum transfer $q=0.64 \mathrm{~nm}^{-1}$. Values of the applied field (in mT): (a) 1500, (b) 290, (c) 100 , and (d) 30 .

high-field susceptbility $\chi$ of nanocrystalline $\mathrm{Fe}_{89} \mathrm{Zr}_{7} \mathrm{~B}_{3} \mathrm{Cu}$ at various temperatures between 5 and $300 \mathrm{~K}$.

It is seen in Figs. 10 and 11 that in the approach-tosaturation regime Nanoperm possesses a relatively large susceptibility (see below), and even at the highest applied field of $\mu_{0} H=7 \mathrm{~T}$ there is a considerable deviation from saturation.

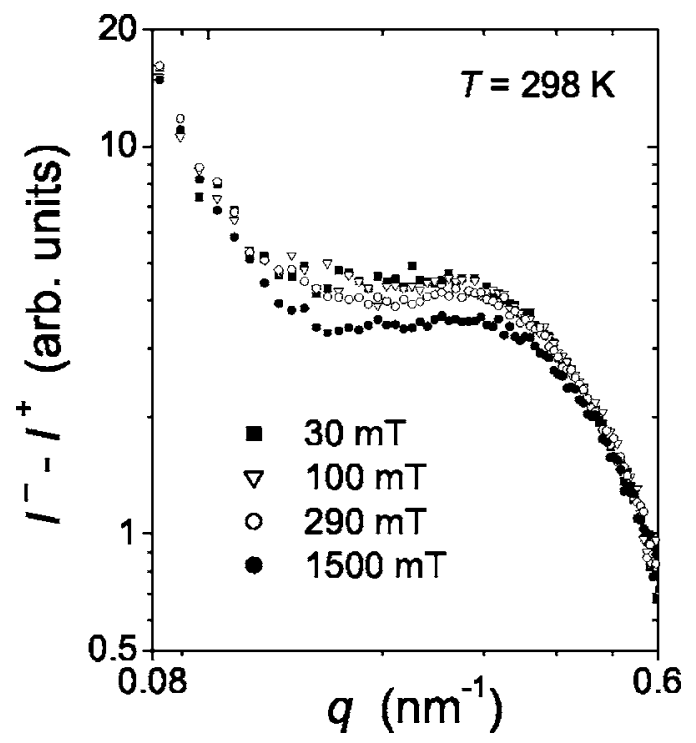

FIG. 9. Radially averaged difference between flipper on and flipper off data, $I^{-}-I^{+}(\log -\log$ scale), as a function of the external magnetic field (see inset). 


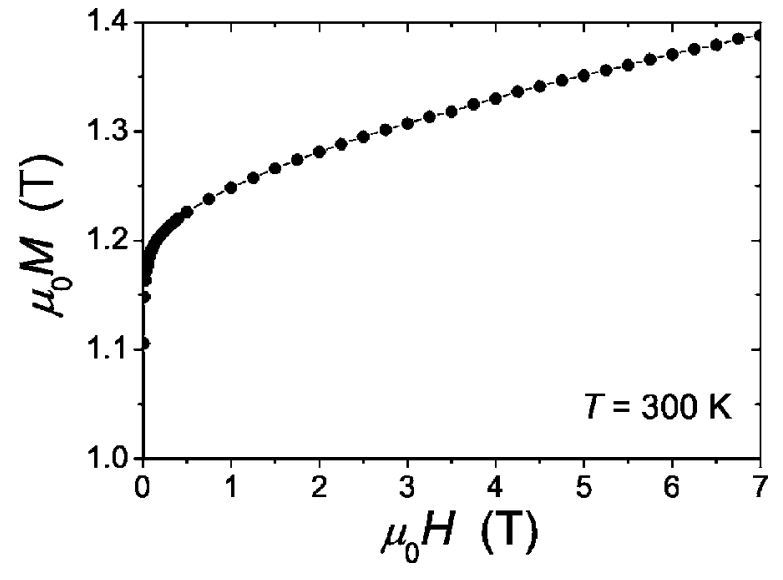

FIG. 10. Room temperature magnetization isotherm of nanocrystalline $\mathrm{Fe}_{89} \mathrm{Zr}_{7} \mathrm{~B}_{3} \mathrm{Cu}$. Line is a guide to the eye.

\section{DISCUSSION}

\section{A. Origin of the scattering contrast}

When the Nanoperm sample is essentially saturated at an external field of $\mu_{0} H=2 \mathrm{~T}$, the total combined nuclear and magnetic SANS cross section $d \Sigma / d \Omega$ [Fig. 2(a)] exhibits a dominant $\sin ^{2} \theta$ behavior, which suggests that the magnetic scattering contrast is much larger than the nuclear SANS signal. ${ }^{77}$ At intermediate applied-field values transversal magnetization fluctuations contribute to the SANS intensity via $d \Sigma_{M} / d \Omega$. Comparison with Eqs. (5) and (6) shows that any angular anisotropy in the unpolarized differenceintensity data (Fig. 3) must be due to terms $m_{y}^{2} \cos ^{2} \theta$ and/or $m_{y} m_{z} \sin \theta \cos \theta$.

By analyzing the SANS data taken with a polarized incident neutron beam (Figs. 8 and 9) it is possible to identify one of these terms as dominant: the difference between data taken with the neutron-spin flipper on and off, $I^{-}-I^{+}$, depends on terms $m_{z} \sin ^{2} \theta$ and $m_{y} \sin \theta \cos \theta$ [compare Eqs.

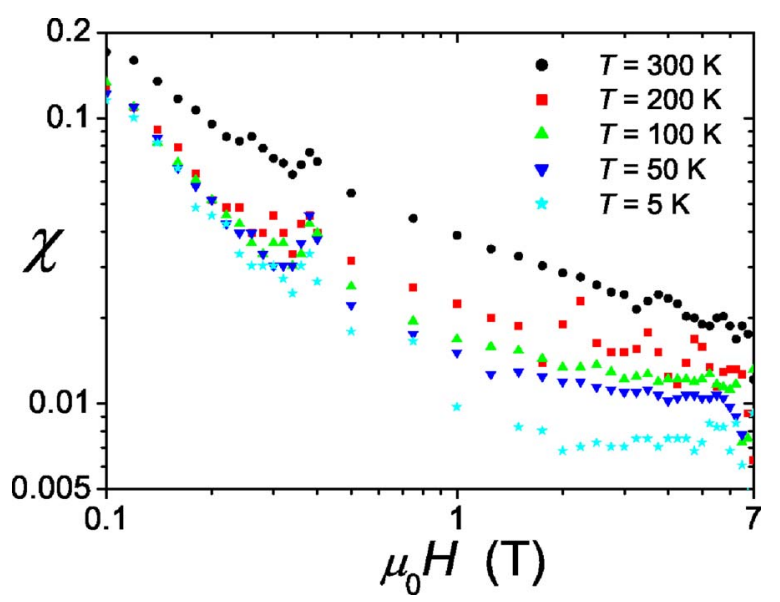

FIG. 11. (Color online) High-field susceptibility $\chi$ of nanocrystalline $\mathrm{Fe}_{89} \mathrm{Zr}_{7} \mathrm{~B}_{3} \mathrm{Cu}$ as a function of the applied magnetic field $H$ and at different temperatures (log-log scale). The quantity $\chi(H)$ at a particular temperature was computed as the numerical derivative of the measured magnetization isotherm ( $\mu_{0} M$ vs $\mu_{0} H$, compare Fig. $10)$.
(5) and (6)]. The observation in Fig. 8 that $I^{-}-I^{+}$is clearly dominated by a $\sin ^{2} \theta$ rather than by a $\sin \theta \cos \theta$ anisotropy shows that throughout the considered high-field regime $(1500-30 \mathrm{mT})$ the transversal spin component is much smaller than the longitudinal component, i.e., $m_{y} \ll m_{z}$. In other words, for our Nanoperm sample and close to saturation, contributions $m_{z} \sin ^{2} \theta$ to the nuclear-magnetic interference term dominate over terms $m_{y} \sin \theta \cos \theta$. With respect to the unpolarized difference-intensity data shown in Fig. 3, this in turn suggests that (for the present sample) the dominating scattering contrast is due to terms proportional to $m_{y} m_{z} \sin \theta \cos \theta$. These terms are linear in the small quantity $m_{y}$, rather than quadratic, as are the terms $m_{y}^{2} \cos ^{2} \theta$ encountered already in studies of elemental nanocrystalline ferromagnets. 56

In support of the above arguments are the results for the radially averaged difference data between the two spin states, which are shown in Fig. 9. Compared to the unpolarized difference-intensity data shown in Fig. $4, I^{-}-I^{+}$is small in magnitude and has a much weaker dependency on the applied magnetic field. This is in accordance with our above conclusion that the prevailing angular anisotropy of $\mathrm{I}^{-}-\mathrm{I}^{+}$ results from the residual scattering cross section, Eq. (5), which, in the small-misalignment approximation, is nearly independent of the applied field. The relative importance (in polarized scattering) of contributions proportional to $m_{y}$ will grow with decreasing field as the transverse spin components are enhanced. Thus the feature at the smallest $q$-values and smallest fields in Fig. 8 might be a fingerprint of a progressively enhanced contribution of terms $m_{y} \sin \theta \cos \theta$ to the scattering.

So far we have discussed the experimental data in relation to the expressions for the spin-dependent nuclear and magnetic SANS cross section derived in Sec. III B. The explicit $\theta$-dependence of the cross section in Eqs. (11) and (12) is a manifestation of the dipolar interaction between the neutron and the individual magnetic moments. As such, the terms $\sin \theta \cos \theta$ in $d \Sigma / d \Omega$ are a priori unrelated to the details of the magnetic microstructure. These details affect the scattering and, in particular, the $\theta$-dependence of $d \Sigma / d \Omega$ via the function $\tilde{M}_{y}(\mathbf{q})$. In view of our finding that $d \Sigma_{M} / d \Omega$ is dominated by the term $\widetilde{M}_{y} \widetilde{M}_{z} \sin \theta \cos \theta$, and of the essentially isotropic character of $\tilde{M}_{z}$, the observed (roughly) $\sin ^{2} \theta \cos ^{2} \theta$ angular dependency of the scattering is therefore an indication that $\tilde{M}_{y}$ depends on the orientation of $\mathbf{q}$ according to $\tilde{M}_{y} \propto \sin \theta \cos \theta$. We shall now discuss the origin of this variation.

\section{B. Dipolar interaction and angular anisotropy}

In Refs. 77 and 78 we have proposed an explanation for the origin of the clover-leaf-shaped anisotropy in the SANS signal in terms of the dipole fields which "decorate" the individual Fe particles: the difference $\Delta M_{S}$ in the saturationmagnetization values between the crystalline Fe particles (higher $M_{S}$ ) and the amorphous magnetic matrix (lower $M_{S}$ ) gives rise to a dipolar stray field which acts as a torque on the magnetic moments and deflects the spins from the per- 


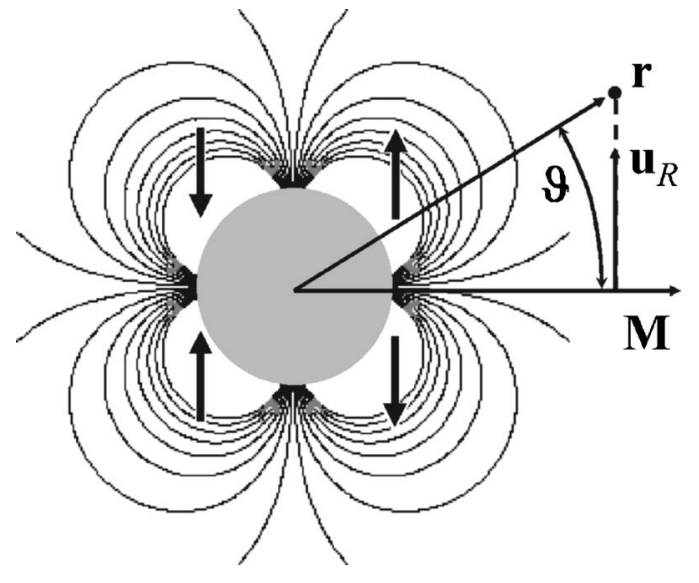

FIG. 12. Sketch illustrating the geometry of the dipole field. Shown is the projection of $\mathbf{H}_{D}^{\perp}$ into a plane containing the magnetization $\mathbf{M}$ of the particle. $\mathbf{H}_{D}^{\perp}$ changes direction at the border between quadrants.

fectly ordered state. For a uniformly magnetized spherical particle of size $D=2 R$, embedded in an infinitely extended coherently saturated matrix, the perpendicular component of the stray field outside of the particle, $\mathbf{H}_{D}^{\perp}(\mathbf{r})$, can be written as $^{105}$

$$
\mathbf{H}_{D}^{\perp}(\mathbf{r})=\Delta M_{S} R^{3} r^{-3} \sin \vartheta \cos \vartheta \mathbf{u}_{R},
$$

where $\vartheta$ is the angle between the position vector $\mathbf{r}(|\mathbf{r}|=r)$ and the direction of saturation, say $\mathbf{e}_{z}$, and $\mathbf{u}_{R}$ $=(\cos \varphi, \sin \varphi, 0)($ compare Fig. 12).

For the Nanoperm sample under study $\mu_{0} \Delta M_{S} \cong 1.5 \mathrm{~T}$, and the resulting dipole field takes on a volume-averaged value of about $270 \mathrm{mT} .^{77,78}$ This value is much larger than the other perturbing fields in this type of material, which originate from magnetocrystalline, uniaxial, or magnetoelastic anisotropy. ${ }^{80,106}$

In the high-field regime, the local transverse magnetization $\mathbf{M}_{D}^{\perp}(\mathbf{r})$ around an individual Fe nanoparticle is to a firstorder approximation proportional to the perturbing dipole field, $\mathbf{M}_{D}^{\perp}(\mathbf{r}) \propto \mathbf{H}_{D}^{\perp}(\mathbf{r})$. Therefore the Fourier transform of the $y$-component of $\mathbf{H}_{D}^{\perp}(\mathbf{r})$ replicates the angular variation of $\tilde{M}_{y}(\mathbf{q})$. In this way it is seen that the predicted effect of the dipole fields on the magnetization agrees with what we inferred from the experimental results (see Sec. V A), namely, $\tilde{M}_{y} \propto \sin \theta \cos \theta$.

While the above considerations suggest maxima in $d \Sigma_{M} / d \Omega$ at angles $\theta= \pm 45^{\circ}$ relative to $\mathbf{H}$, the experimental difference-intensity data in Figs. 3 and 5 peak at $\theta$ $\cong \pm 30^{\circ}-40^{\circ}$, i.e., at an angle $\theta$ which is significantly less than $45^{\circ}$. This observation may be explained qualitatively by comparison with Eq. (12) for the spin-misalignment scattering cross section: the $d \Sigma_{M} / d \Omega$ that is due to the dipole-field effect presumably leads to $d \Sigma_{M} / d \Omega \propto \hat{M}_{y} \widetilde{M}_{z} \sin ^{2} \theta \cos ^{2} \theta$, where $\hat{M}_{y}$ denotes the angular independent part of $\tilde{M}_{y}$, i.e., we write $\widetilde{M}_{y}(\mathbf{q})=\hat{M}_{y}(q) \sin \theta \cos \theta$ (see discussion in the preceding paragraph). The terms in $d \Sigma_{M} / d \Omega$ which are quadratic in the transversal spin components then result in
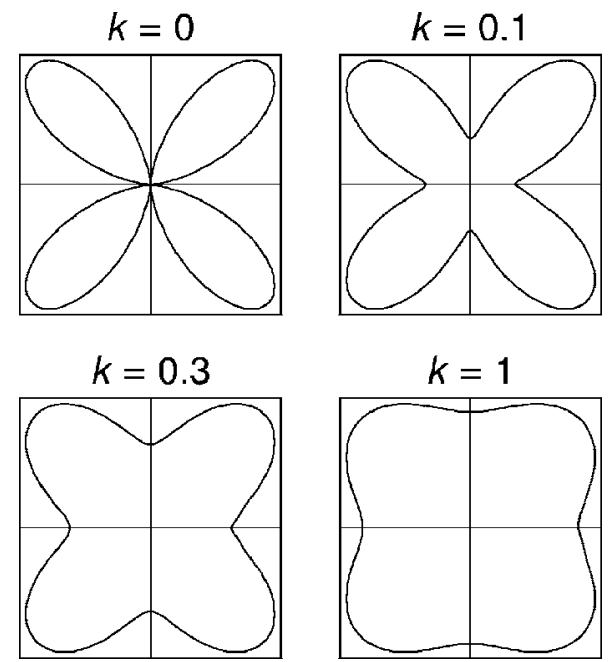

FIG. 13. Polar plots of the function $\sin ^{2} \theta \cos ^{2} \theta+k(1$ $+\sin ^{2} \theta \cos ^{4} \theta$ ) for different values of the parameter $k$, as indicated.

$d \Sigma_{M} / d \Omega \propto \hat{M}_{y}^{2}\left(\tilde{M}_{x}^{2} / \hat{M}_{y}^{2}+\sin ^{2} \theta \cos ^{4} \theta\right)$. Assuming that $\tilde{M}_{z}$ is isotropic and that $\tilde{M}_{x} \cong \hat{M}_{y}$, the combination of the above results may then lead to

$$
\frac{d \Sigma_{M}}{d \Omega} \propto \sin ^{2} \theta \cos ^{2} \theta+k\left(1+\sin ^{2} \theta \cos ^{4} \theta\right),
$$

where the magnetic-field-dependent parameter $k$ accounts for the relative contribution of the $\left(1+\sin ^{2} \theta \cos ^{4} \theta\right)$ term to the overall anisotropy of the difference-intensity data; by construction, this term increases as the transverse spin components become enhanced with decreasing field.

Figure 13 depicts polar plots of the function $\sin ^{2} \theta \cos ^{2} \theta$ $+k\left(1+\sin ^{2} \theta \cos ^{4} \theta\right)$ for different values of the parameter $k$. These graphs qualitatively reproduce the field-dependent difference-intensity data shown in Fig. 3.

Since the term $\left(1+\sin ^{2} \theta \cos ^{4} \theta\right)$ depends quadratically on $M_{y}$, it is, at large applied fields, negligible compared to the $\sin ^{2} \theta \cos ^{2} \theta$ term, which depends linearly on $M_{y}$. On decreasing the field, the relative importance of the (1 $\left.+\sin ^{2} \theta \cos ^{4} \theta\right)$ term increases, and, consequently, the angular anisotropy of the SANS pattern changes, as can be seen qualitatively in Fig. 13.

Whether or not dipole fields constitute the dominant perturbing field in a particular nanocomposite ferromagnet may depend on a variety of parameters such as the applied magnetic field, volume fraction of particles, particle shape and particle-size distribution, temperature, and contrast in magnetization magnitude between the phases. In this context we note that SANS investigations on Nanoperm with a higher volume fraction of particles, $x_{C}=70 \%$, and on Vitroperm $\left(\mathrm{Fe}_{73} \mathrm{Si}_{16} \mathrm{~B}_{7} \mathrm{Nb}_{3} \mathrm{Cu}\right)$ (Ref. 75) with $\mathrm{FeSi}$ crystallites of reduced magnetization did not show the clover-leaf-shaped pattern. Conceivably, the crystallites are less separated by the matrix phase in these systems with a higher crystalline volume fraction. The dipolar effect is expected to be less important when crystallites form contiguous chains or clusters of 
grains joined by grain boundaries rather than separated by the matrix phase.

\section{Interparticle correlations and temperature dependence}

Based on the average Fe particle size, $D=12 \mathrm{~nm}$, and on the value of the crystalline volume fraction, $x_{C}=40 \%$, we estimate the mean thickness $\delta$ of the matrix layer at about $4 \mathrm{~nm}$ using $\delta=D\left(x_{C}^{-1 / 3}-1\right)$ (Ref. 107). Therefore dipole fields which emerge from neighboring particles will not only overlap, but they will also extend, at least partly, through the neighboring crystallites. Closer inspection of the magneticfield dependence of the unpolarized difference-intensity data in Figs. 4(a) and 4(b) suggests that the characteristic wavelength of the dipole-field-induced spin misalignment varies as a function of the applied magnetic field: the region with the largest curvature in the difference signal shifts to larger $q$-values as the field is increased, which indicates that longwavelength fluctuations of the magnetization are progressively suppressed by the applied field. In particular, the observation that significant spin-misalignment scattering exists even at the smallest $q$-values $\left(2 \pi / 0.08 \mathrm{~nm}^{-1} \cong 80 \mathrm{~nm}\right)$ suggests the existence of magnetic dipolar correlations on length scales even much larger than the mean particle size of $D$ $=12 \mathrm{~nm}$. This assessment is further supported by the results of the temperature-dependent data (Fig. 5), where it can be clearly seen that the characteristic angular anisotropy of the dipole-field effect is also visible at the smallest $q$-values.

The temperature-dependent data (Figs. 5 and 6) additionally demonstrate that the long-range correlated spin misalignment is present in the spin system even up to several hundred degrees Kelvin above the Curie point of the amorphous intergranular matrix phase $\left(T_{C}^{\mathrm{am}}=345 \mathrm{~K}\right)$. Furthermore, the SANS curves at different temperatures in Fig. 6 seem to be "self-similar," i.e., no temperature-dependent feature in the $q$-dependency can be detected. This observation suggests that the characteristic length scale on which the dipole-field-induced spin misalignment in our Nanoperm sample evolves is independent of temperature. The characteristic wavelength of the spin disorder was estimated above by inspection of the field dependence of the radially averaged difference-intensity data (Fig. 4) and was found to be of the order of at least $80 \mathrm{~nm}$.

Assuming that the spontaneous magnetization of the $\mathrm{Fe}$ particles varies only slightly between $T_{C}^{\mathrm{am}}$ and the highest available temperature, $T=693 \mathrm{~K}$, as is true for $\mathrm{Fe}$ single crystals, ${ }^{128}$ it is clear that the magnitude of the perturbing dipole field remains almost constant over this temperature range, since $\Delta M_{S}$ is essentially given by $M_{S}$ of the Fe nanoparticles [compare Fig. 7(b)]. As a consequence of a reduced susceptibility, we expect the response of the paramagnetic matrix to the dipole field to be smaller above $T_{C}^{\mathrm{am}}$ than below $T_{C}^{\mathrm{am}}$. Hence the disordering effect of the dipole field is smaller above $T_{C}^{\mathrm{am}}$, which explains the decrease of the spinmisalignment scattering signal with increasing temperature, as can be seen in Fig. 6.

The findings below suggest that there exists some degree of long-range correlation between the $\mathrm{Fe}$ particles, even when the matrix is paramagnetic. That the coupling between the Fe particles is not completely interrupted above $T_{C}^{\mathrm{am}}$ has also been found previously by magnetization and Mössbauer studies on Fe-based nanocomposite alloys (see, e.g., Refs. 80,82 , and $108-110$ and references therein). Moreover, investigations with Kerr microscopy on Vitroperm-type alloys with a large induced uniaxial anisotropy reveal macroscopic domain patterns well above $T_{C}^{\mathrm{am}} \cdot{ }^{111}$

However, the nature of the coupling mechanism is still a matter of debate. ${ }^{12}$ While Hernando and Kulik ${ }^{108}$ suggested that the coupling between the Fe particles through the paramagnetic matrix is accomplished by exchange polarization, Herzer ${ }^{113}$ showed that the experimental results may also be explained by assuming pure dipolar coupling between the particles. Even in amorphous reentrant systems dipolar interactions have been linked to long-range ferromagnetic order. ${ }^{114}$ Due to the presence of conduction electrons in the metallic intergranular layer, a third mechanism of the RKKYtype is in principle also possible. Recent calculations by Altbir et al. ${ }^{115}$ and by Skomski ${ }^{116}$ suggest, however, the absence of the RKKY interaction mechanism for well-separated magnetic particles (larger than about $1 \mathrm{~nm}$ ) in a nonmagnetic metallic matrix due to the dominance of the magnetostatic interaction. Although the present data may favor the dominance of the dipolar interaction, the existence of exchange coupling through the paramagnetic intergranular layer cannot be ruled out.

\section{Influence on macroscopic parameters}

It may be expected that the dipole-field effect, besides giving rise to the characteristic anisotropy in the SANS pattern, will also affect the macroscopic magnetic behavior, in particular, the high-field susceptibility. Indeed, the magnetization data shown in Figs. 10 and 11 exhibit a relatively large susceptibility up to the highest applied field of $\mu_{0} H$ $=7 \mathrm{~T}$. The values of $\chi$ in Fig. 11 are relatively large compared to the "usual" contributions such as the Pauli spin susceptibility $\left(\chi \sim 10^{-5}-10^{-6}\right)$ or to the susceptibility due to the so-called paraeffect. ${ }^{117}$ The field dependence of the latter at $T=300 \mathrm{~K}$ can be estimated based on the materials parameters spin-wave stiffness constant $D, g$-factor $g$, and zerotemperature saturation magnetization $M_{S}^{0}$. Using $D$ $=141 \mathrm{meV} \AA^{2}, g=2.10$, and $M_{S}^{0}=1.7 \mathrm{~T}$, we find, however, that the paraeffect gives rise to a significantly lower susceptibility which varies between $\chi \cong 0.01$ at $0.1 \mathrm{~T}$ and $\chi$ $\cong 0.002$ at $7 \mathrm{~T} .{ }^{117,118}$ Likewise, micromagnetic computations by Kronmüller ${ }^{119}$ indicate that the magnitude of the experimental susceptibility can also not be explained by the dipole fields. Possible origins for the increased high-field susceptibility in our Nanoperm sample might be related to the existence of ferrimagnetic or antiferromagnetic couplings within the Fe-rich amorphous matrix, ${ }^{118,120,121}$ spin canting, or to the presence of weak itinerant ferromagnetism. ${ }^{122}$

\section{SUMMARY AND CONCLUDING REMARKS}

We have presented the results of the magnetic-field, temperature, and neutron-polarization dependence of dipolefield-induced spin-misalignment scattering in the soft mag- 
netic two-phase nanocomposite $\mathrm{Fe}_{89} \mathrm{Zr}_{7} \mathrm{~B}_{3} \mathrm{Cu}$ (Nanoperm). The jump $\Delta M_{S}$ in the saturation magnetization at the interface between the iron nanoparticles and the amorphous magnetic matrix gives rise to a dipolar stray field $\mathbf{H}_{D}$ which acts as a torque on the magnetic moments and, thereby, induces spin disorder at the nanoscale. The dipole-field-induced spin disorder gives rise to a clover-leaf-shaped angular anisotropy in the magnetic small-angle neutron scattering (SANS) cross section with maximum intensity at angles $\theta \cong \pm 30^{\circ}-40^{\circ}$ relative to the direction of the applied magnetic field $\mathbf{H}$. The clover-leaf-shaped pattern is observed between room temperature and $T=693 \mathrm{~K}$, i.e., several hundred degrees Kelvin above the decoupling temperature of the intergranular amorphous matrix phase $\left(T_{C}^{\mathrm{am}}=345 \mathrm{~K}\right)$. From field-dependent SANS data we conclude that the range of the dipolar correlations extends to length scales of at least $80 \mathrm{~nm}$, considerably larger than the mean iron-particle size of $D=12 \mathrm{~nm}$. We have derived expressions for the spin-dependent nuclear and magnetic small-angle neutron scattering cross section $d \Sigma / d \Omega$ of a multicomponent and multiphase nanocrystalline ferromagnet. The cross section $d \Sigma / d \Omega$ contains anisotropic magnetic contributions proportional to $\sin ^{2} \theta, \cos ^{2} \theta$, and $\sin \theta \cos \theta$, which are a direct manifestation of the dipolar character of magnetic neutron scattering. In particular, the experimentally observed clover-leaf-shaped anisotropy can be explained by terms $m_{y} m_{z} \sin \theta \cos \theta$, which yield maximum intensity at angles $\theta= \pm 45^{\circ}$ relative to $\mathbf{H}$. As a consequence of the fact that within the magnetic-field regime studied $(30-1500 \mathrm{mT})$ the transversal spin component $m_{y}$ is much smaller than the longitudinal component $m_{z}$, the difference in the SANS intensities with the neutron-spin flipper on and off, $I^{-}-I^{+}$, exhibits a dominating $\sin ^{2} \theta$ anisotropy. It is expected that dipole-field-induced spin disorder has no significant influence on the high-field susceptibility.

In contrast to our previous SANS work on single-phase and single-component nanocrystalline ferromagnets, ${ }^{56}$ contributions $\tilde{M}_{y} \tilde{M}_{z} \sin \theta \cos \theta$ to $d \Sigma_{M} / d \Omega$ are found to dominate the scattering (at intermediate field values) in the present study on the composite material Nanoperm. For homogeneous elemental ferromagnets such as nanoscaled $\mathrm{Ni}$ or Co, $d \Sigma_{M} / d \Omega$ can be computed in the approach-to-saturation regime using the continuum theory of micromagnetics. ${ }^{123-125}$ Note that due to the nonlinear nature of the underlying par- tial integrodifferential equations of micromagnetics, certain approximations, such as small misalignment of magnetic moments, are generally inevitable in order to arrive at closed-form expressions. As we have outlined in Refs. 56, 59 , and 60 , when the micromagnetic approach is followed, one is able to determine the volume-averaged magnetic interaction parameters of a nanocrystalline ferromagnet such as the exchange-stiffness constant, magnetic anisotropy field, magnetostatic stray field (due to $\boldsymbol{\nabla} \cdot \mathbf{M} \neq 0$ ), and it even becomes possible to compare micromagnetic predictions for the exchange length of the magnetic field with experimental SANS data. However, the straightforward analytical calculation of magnetization profiles (and ensuing $d \Sigma_{M} / d \Omega$ ) in inhomogeneous nanocomposites entails, even close to saturation, great mathematical difficulties, ${ }^{126}$ since additional boundary conditions at the interface between particles and matrix have to be taken into account.

As a final comment we would like to note that the cloverleaf-shaped angular anisotropy might be overlooked in experiments if one measures the scattering cross section only at saturation and at zero applied magnetic field, which corresponds to the situation where the vast majority of magnetic SANS studies have been performed to date. Measurements at intermediate field values are therefore necessary, and the subtraction of the $\sin ^{2} \theta$ scattering at saturation is helpful, in order to reveal the clover-leaf-shaped anisotropy.

\section{ACKNOWLEDGMENTS}

This research has been partly financed by the European Commission under the 6th Framework Programme through the Key Action "Strengthening the European Research Area, Research Infrastructures" (Contract No. RII3-CT-2003505925). The work is based on experiments performed at the Swiss spallation neutron source SINQ, Paul Scherrer Institute, Villigen, Switzerland. Support by Landesstiftung Baden-Württemberg, and by a MIUR postgraduate bursary (C.V.) is gratefully acknowledged. We thank H. Eckerlebe and G. Kozik for help with the SANS experiments, J. Schmauch, R. Karos, S. Oldenbürger, and J. Markmann for TEM, x-ray, and DSC measurements. A critical reading of the manuscript by R. Birringer, A. Tschöpe, S. N. Kaul, and H. B. Stuhrmann is gratefully acknowledged. A.M. thanks Monash University for its hospitality.
*Corresponding author. FAX: $++49-(0) 681-302-5222$; electronic address: andreas.michels@mx.uni-saarland.de

${ }^{1}$ R. Skomski, J. Phys.: Condens. Matter 15, R841 (2003).

${ }^{2}$ A. Hubert and R. Schäfer, Magnetic Domains (Springer, Berlin, 1998).

${ }^{3}$ U. Hartmann, Annu. Rev. Mater. Sci. 29, 53 (1999).

${ }^{4}$ R. Wiesendanger, Scanning Probe Microscopy and Spectroscopy

(Cambridge University Press, Cambridge, 1994).

${ }^{5}$ M. Bode, Rep. Prog. Phys. 66, 523 (2003).

${ }^{6}$ E. Bauer, J. Phys.: Condens. Matter 13, 11391 (2001).

${ }^{7}$ D. J. Cebula, S. W. Charles, and J. Popplewell, Colloid Polym.
Sci. 259, 395 (1981).

${ }^{8}$ R. Pynn, J. B. Hayter, and S. W. Charles, Phys. Rev. Lett. 51, 710 (1983).

${ }^{9}$ R. Rosman, J. J. M. Janssen, and M. Th. Rekveldt, J. Appl. Phys. 67, 3072 (1990).

${ }^{10}$ S. Itoh, Y. Endoh, and S. W. Charles, J. Magn. Magn. Mater. 111, 56 (1992).

${ }^{11}$ F. Gazeau, E. Dubois, J.-C. Bacri, F. Boué, A. Cebers, and R. Perzynski, Phys. Rev. E 65, 031403 (2002).

${ }^{12}$ A. Wiedenmann, in Magnetically Controllable Fluids and Their Applications, edited by S. Odenbach, Lecture Notes in Physics 
Vol. 594 (Springer, Berlin, 2002), pp. 33-58.

${ }^{13}$ A. Wiedenmann, A. Hoell, M. Kammel, and P. Boesecke, Phys. Rev. E 68, 031203 (2003).

${ }^{14}$ M. Ernst, J. Schelten, and W. Schmatz, Phys. Status Solidi A 7, 469 (1971).

${ }^{15}$ M. Sato and K. Hirakawa, J. Phys. Soc. Jpn. 39, 1467 (1975).

${ }^{16}$ R. Cywinski, J. G. Booth, and B. D. Rainford, J. Phys. F: Met. Phys. 7, 2567 (1977).

${ }^{17}$ G. Abersfelder, K. Noack, K. Stierstadt, J. Schelten, and W. Schmatz, Philos. Mag. B 41, 519 (1980).

${ }^{18}$ P. Kournettas, K. Stierstadt, and D. Schwahn, Philos. Mag. B 51, 381 (1985).

${ }^{19}$ A. C. Nunes, J. Appl. Crystallogr. 21, 129 (1988).

${ }^{20}$ P. Ancrenaz and C. Servant, J. Phys. I 2, 1113 (1992).

${ }^{21}$ J. R. Childress, C. L. Chien, J. J. Rhyne, and R. W. Erwin, J. Magn. Magn. Mater. 104-107, 1585 (1992).

${ }^{22}$ W. Wagner, Acta Metall. Mater. 38, 2711 (1990).

${ }^{23}$ C. Bellouard, I. Mirebeau, and M. Hennion, Phys. Rev. B 53, 5570 (1996).

${ }^{24}$ Y. J. Wang, R. Kampmann, and R. Wagner, Physica B 234-236, 992 (1997).

${ }^{25}$ U. Lembke, A. Hoell, R. Kranold, R. Müller, W. Schüppel, G. Goerigk, R. Gilles, and A. Wiedenmann, J. Appl. Phys. 85, 2279 (1999).

${ }^{26}$ A. Michels and J. Weissmüller, Eur. Phys. J. B 26, 57 (2002).

${ }^{27}$ T. Thomson, S. L. Lee, M. F. Toney, C. D. Dewhurst, F. Y. Ogrin, C. J. Oates, and S. Sun, Phys. Rev. B 72, 064441 (2005).

${ }^{28}$ Y. Ijiri, C. V. Kelly, J. A. Borchers, J. J. Rhyne, D. F. Farrell, and S. A. Majetich, Appl. Phys. Lett. 86, 243102 (2005).

${ }^{29}$ J. Suzuki, K. Takei, Y. Maeda, and Y. Morii, J. Magn. Magn. Mater. 184, 116 (1998).

${ }^{30}$ M. F. Toney, K. A. Rubin, S.-M. Choi, and C. J. Glinka, Appl. Phys. Lett. 82, 3050 (2003).

${ }^{31}$ M. P. Wismayer, S. L. Lee, T. Thomson, F. Y. Ogrin, C. D. Dewhurst, S. M. Weekes, and R. Cubitt, J. Appl. Phys. 99, 08E707 (2006).

${ }^{32}$ F. Y. Ogrin, S. L. Lee, M. Wismayer, T. Thomson, C. D. Dewhurst, R. Cubitt, and S. M. Weekes, J. Appl. Phys. 99, 08G912 (2006).

${ }^{33}$ M. Viret, F. Ott, J. P. Renard, H. Glättli, L. Pinsard-Gaudart, and A. Revcolevschi, Phys. Rev. Lett. 93, 217402 (2004).

${ }^{34}$ N. Veglio, F. J. Bermejo, J. Gutierrez, J. M. Barandiarán, A. Peña, M. A. González, P. P. Romano, and C. Mondelli, Phys. Rev. B 71, 212402 (2005).

${ }^{35}$ D. Saurel, A. Brûlet, A. Heinemann, C. Martin, S. Mercone, and C. Simon, Phys. Rev. B 73, 094438 (2006).

${ }^{36}$ A. P. Murani, S. Roth, P. Radhakrishna, B. D. Rainford, B. R. Coles, K. Ibel, G. Goeltz, and F. Mezei, J. Phys. F: Met. Phys. 6, 425 (1976).

${ }^{37}$ G. Aeppli, S. M. Shapiro, R. J. Birgeneau, and H. S. Chen, Phys. Rev. B 28, 5160 (1983).

${ }^{38}$ O. Moze, E. J. Lindley, B. D. Rainford, and D. MckPaul, J. Magn. Magn. Mater. 53, 167 (1985).

${ }^{39}$ T. Oku, E. Ohta, T. Sato, M. Furusaka, M. Imai, and Y. Matsushita, J. Magn. Magn. Mater. 188, 291 (1998).

${ }^{40}$ B. Schönfeld, O. Paris, G. Kostorz, and J. S. Pedersen, J. Phys.: Condens. Matter 10, 8395 (1998).

${ }^{41}$ R. García Calderón, L. Fernández Barquín, S. N. Kaul, J. C. Gómez Sal, P. Gorria, J. S. Pedersen, and R. Heenan, Phys. Rev. B 71, 134413 (2005).
${ }^{42}$ S. Komura, G. Lippmann, and W. Schmatz, J. Appl. Crystallogr. 7, 233 (1974)

${ }^{43}$ S. V. Grigoriev, S. A. Klimko, W. H. Kraan, S. V. Maleyev, A. I. Okorokov, M. Th. Rekveldt, and V. V. Runov, Phys. Rev. B 64, 094426 (2001).

${ }^{44}$ O. Schaerpf, H. Vehoff, and C. Schwink, Int. J. Magn. 5, 223 (1973).

${ }^{45}$ G. Göltz, H. Kronmüller, A. Seeger, H. Scheuer, and W. Schmatz, Philos. Mag. A 54, 213 (1986).

${ }^{46}$ A. I. Okorokov, S. V. Grigoriev, Y. O. Chetverikov, R. Georgii, P. Böni, H. Eckerlebe, K. Pranzas, and B. Roessli, Physica B 350, 323(E) (2004).

${ }^{47}$ M. Weber, W. Knoll, and S. Steeb, J. Appl. Crystallogr. 11, 638 (1978).

${ }^{48}$ R. Coppola, R. Kampmann, M. Magnani, and P. Staron, Acta Mater. 46, 5447 (1998).

${ }^{49}$ M. Bischof, S. Erlach, P. Staron, H. Leitner, C. Scheu, and H. Clemens, Z. Metallkd. 96, 1074 (2005).

${ }^{50}$ B. van den Brandt, H. Glättli, I. Grillo, P. Hautle, H. Jouve, J. Kohlbrecher, J. A. Konter, E. Leymarie, S. Mango, R. P. May, A. Michels, H. B. Stuhrmann, and O. Zimmer, Eur. Phys. J. B 49, 157 (2006).

${ }^{51}$ J. Weissmüller, A. Michels, D. Michels, A. Wiedenmann, C. E. Krill III, H. M. Sauer, and R. Birringer, Phys. Rev. B 69, 054402 (2004).

${ }^{52}$ W. Wagner, A. Wiedenmann, W. Petry, A. Geibel, and H. Gleiter, J. Mater. Res. 6, 2305 (1991).

${ }^{53}$ J. F. Löffler, H. B. Braun, and W. Wagner, Phys. Rev. Lett. 85, 1990 (2000).

${ }^{54}$ J. Weissmüller and A. Michels, Phys. Rev. Lett. 87, 149701 (2001).

55 J. F. Löffler, H. B. Braun, and W. Wagner, Phys. Rev. Lett. 87, 149702 (2001).

${ }^{56}$ J. Weissmüller, A. Michels, J. G. Barker, A. Wiedenmann, U. Erb, and R. D. Shull, Phys. Rev. B 63, 214414 (2001).

${ }^{57}$ R. Przeniosło, R. Winter, H. Natter, M. Schmelzer, R. Hempelmann, and W. Wagner, Phys. Rev. B 63, 054408 (2001).

${ }^{58}$ R. Przeniosło, J. Wagner, H. Natter, R. Hempelmann, and W. Wagner, J. Alloys Compd. 328, 259 (2001).

${ }^{59}$ A. Michels, J. Weissmüller, and R. Birringer, Eur. Phys. J. B 29, 533 (2002).

${ }^{60}$ A. Michels, R. N. Viswanath, J. G. Barker, R. Birringer, and J. Weissmüller, Phys. Rev. Lett. 91, 267204 (2003).

${ }^{61}$ J. F. Löffler, H. B. Braun, W. Wagner, G. Kostorz, and A. Wiedenmann, Phys. Rev. B 71, 134410 (2005).

${ }^{62}$ A. Bracchi, K. Samwer, S. Schneider, and J. F. Löffler, Appl. Phys. Lett. 82, 721 (2003).

${ }^{63}$ E. García-Matres, A. Wiedenmann, G. Kumar, J. Eckert, H. Hermann, and L. Schultz, Physica B 350, 315(E) (2004).

${ }^{64}$ M. Ohnuma, J. Suzuki, S. Funahashi, T. Ishigaki, H. Kuwano, and Y. Hamaguchi, Mater. Trans., JIM 36, 918 (1995).

${ }^{65}$ A. Deriu, F. Malizia, F. Ronconi, M. Vittori-Antisari, and S. M. King, J. Appl. Phys. 79, 2296 (1996).

${ }^{66}$ H. N. Frase, B. Fultz, S. Spooner, and J. L. Robertson, J. Appl. Phys. 85, 7097 (1999).

${ }^{67}$ J. Kohlbrecher, A. Wiedenmann, and H. Wollenberger, Z. Phys. B: Condens. Matter 104, 1 (1997).

${ }^{68}$ A. Danzig, A. Wiedenmann, and N. Mattern, J. Phys.: Condens. Matter 10, 5267 (1998).

${ }^{69}$ I. V. Golosovsky, G. Török, E. I. Maltzev, and I. Mirebeau, 
Physica B 276-278, 918 (2000).

${ }^{70}$ A. Heinemann, H. Hermann, A. Wiedenmann, N. Mattern, and K. Wetzig, J. Appl. Crystallogr. 33, 1386 (2000).

${ }^{71}$ H. Hermann, A. Heinemann, N. Mattern, and A. Wiedenmann, Europhys. Lett. 51, 127 (2000).

${ }^{72}$ J. Marcin, A. Wiedenmann, and I. Škorvánek, Physica B 276278, 870 (2000).

${ }^{73}$ M. Ohnuma, K. Hono, S. Linderoth, J. S. Pedersen, Y. Yoshizawa, and H. Onodera, Acta Mater. 48, 4783 (2000).

${ }^{74}$ E. Agosti, O. Moze, J. Cadogan, K. Suzuki, A. Heinemann, and A. Hoell, Mater. Res. Soc. Symp. Proc. 746, R3.9.1 (2003).

${ }^{75}$ A. Michels, R. N. Viswanath, and J. Weissmüller, Europhys. Lett. 64, 43 (2003b).

${ }^{76}$ A. Grob, S. Saranu, U. Herr, A. Michels, R. N. Viswanath, and J. Weissmüller, Phys. Status Solidi A 201, 3354 (2004).

${ }^{77}$ A. Michels, C. Vecchini, O. Moze, K. Suzuki, J. M. Cadogan, P. K. Pranzas, and J. Weissmüller, Europhys. Lett. 72, 249 (2005).

${ }^{78}$ C. Vecchini, O. Moze, K. Suzuki, P. K. Pranzas, J. Weissmüller, and A. Michels, Appl. Phys. Lett. 87, 202509 (2005).

${ }^{79}$ W. Schmatz, T. Springer, J. Schelten, and K. Ibel, J. Appl. Crystallogr. 7, 96 (1974).

${ }^{80} \mathrm{G}$. Herzer, in Handbook of Magnetic Materials, edited by K. H. J. Buschow (Elsevier, Amsterdam, 1997), Vol. 10, pp. 415-462.

${ }^{81}$ K. Suzuki and G. Herzer, in Advanced Magnetic Nanostructures, edited by D. Sellmyer and R. Skomski (Springer, New York, 2006), pp. 365-401.

${ }^{82}$ K. Suzuki and J. M. Cadogan, Phys. Rev. B 58, 2730 (1998).

${ }^{83}$ K.-Y. He, J. Zhi, L.-Z. Cheng, and M.-L. Sui, Mater. Sci. Eng., A 181-182, 880 (1994).

${ }^{84}$ T. Kemény, D. Kaptás, J. Balogh, L. F. Kiss, T. Pusztai, and I. Vincze, J. Phys.: Condens. Matter 11, 2841 (1999).

${ }^{85}$ L. K. Varga, L. Novák, and F. Mazaleyrat, J. Magn. Magn. Mater. 210, L25 (2000).

${ }^{86}$ Y. F. Li, D. X. Chen, M. Vazquez, and A. Hernando, J. Phys. D 35, 508 (2002).

${ }^{87}$ Y. Gao, D. Shindo, T. Bitoh, and A. Makino, Phys. Rev. B 67, 172409 (2003).

${ }^{88}$ V. Franco, C. F. Conde, A. Conde, and L. F. Kiss, Phys. Rev. B 72, 174424 (2005).

${ }^{89}$ J. M. Vargas, W. C. Nunes, L. M. Socolovsky, M. Knobel, and D. Zanchet, Phys. Rev. B 72, 184428 (2005).

${ }^{90}$ The angular anisotropy of the SANS intensity was previously denoted as "fourfold" (Refs. 77 and 78). However, the symmetry on the two-dimensional SANS detector is not fourfold (but twofold), since a rotation of the intensity distribution by $90^{\circ}$ will not result in the same pattern. Therefore, in the following, we prefer the term "clover-leaf-shaped" instead of fourfold.

${ }^{91} \mathrm{M}$. Bischof (private communication).

${ }^{92}$ J. Kohlbrecher and W. Wagner, J. Appl. Crystallogr. 33, 804 (2000).

${ }^{93}$ See http://genf.gkss.de

${ }^{94}$ U. Keiderling, Appl. Phys. A: Mater. Sci. Process. 74, S1455 (2002).

95 J. Schelten and W. Schmatz, J. Appl. Crystallogr. 13, 385 (1980).

${ }^{96}$ R. M. Moon, T. Riste, and W. C. Koehler, Phys. Rev. 181, 920 (1969)
${ }^{97}$ S. W. Lovesey, Theory of Neutron Scattering from Condensed Matter (Clarendon Press, Oxford, 1986), Vol. II.

${ }^{98}$ W. G. Williams, Polarized Neutrons (Clarendon Press, Oxford, 1988).

${ }^{99}$ T. J. Hicks, Magnetism in Disorder (Clarendon Press, Oxford, 1995).

${ }^{100}$ J. Weissmüller, R. D. McMichael, A. Michels, and R. D. Shull, J. Res. Natl. Inst. Stand. Technol. 104, 261 (1999).

${ }^{101}$ O. Halpern and M. H. Johnson, Phys. Rev. 55, 898 (1939).

${ }^{102}$ M. Blume, Phys. Rev. 130, 1670 (1963).

${ }^{103}$ J. Rossat-Mignod, in Methods of Experimental Physics-Neutron Scattering, edited by K. Sköld and D. L. Price (Academic Press, Orlando, 1987), Vol. 23, Part C, pp. 69-157.

${ }^{104}$ A. Aharoni, J. Appl. Phys. 83, 3432 (1998).

${ }^{105}$ J. D. Jackson, Classical Electrodynamics, 2nd ed. (Walter de Gruyter, Berlin, 1983), Chap. 5.

${ }^{106}$ G. Herzer, J. Magn. Magn. Mater. 294, 99 (2005).

${ }^{107}$ G. Herzer, IEEE Trans. Magn. 25, 3327 (1989).

${ }^{108}$ A. Hernando and T. Kulik, Phys. Rev. B 49, 7064 (1994).

${ }^{109}$ A. Ślawska-Waniewska, P. Nowicki, H. K. Lachowicz, P. Gorria, J. M. Barandiarán, and A. Hernando, Phys. Rev. B 50, 6465 (1994).

${ }^{110}$ J. S. Garitaonandia, D. S. Schmool, and J. M. Barandiarán, Phys. Rev. B 58, 12147 (1998).

${ }^{111}$ S. Flohrer, R. Schäfer, C. Polak, and G. Herzer, Acta Mater. 53, 2937 (2005).

${ }^{112}$ G. Herzer and L. K. Varga, J. Magn. Magn. Mater. 215-216, 506 (2000).

${ }^{113}$ G. Herzer, Scr. Metall. Mater. 33, 1741 (1995).

${ }^{114}$ S. Srinath and S. N. Kaul, Europhys. Lett. 51, 441 (2000).

${ }^{115}$ D. Altbir, J. d'Albuquerque e Castro, and P. Vargas, Phys. Rev. B 54, R6823 (1996).

${ }^{116}$ R. Skomski, Europhys. Lett. 48, 455 (1999).

${ }^{117}$ T. Holstein and H. Primakoff, Phys. Rev. 58, 1098 (1940).

${ }^{118}$ A. Neuweiler, B. Hofmann, and H. Kronmüller, J. Magn. Magn. Mater. 153, 28 (1996).

${ }^{119}$ H. Kronmüller, Z. Phys. 168, 478 (1962).

${ }^{120}$ A. Arrott, J. Appl. Phys. 34, 1108 (1963).

${ }^{121}$ H. Kronmüller, V. A. Ignatchenko, and A. Forkl, J. Magn. Magn. Mater. 134, 68 (1994).

${ }^{122}$ S. N. Kaul and P. D. Babu, J. Phys.: Condens. Matter 10, 1563 (1998).

${ }^{123}$ W. F. Brown, Jr., Micromagnetics (Interscience Publishers, New York, 1963).

${ }^{124}$ A. Aharoni, Introduction to the Theory of Ferromagnetism 2nd ed. (Clarendon Press, Oxford, 1966).

${ }^{125} \mathrm{~K}$. Kronmüller and M. Fähnle, Micromagnetism and the Microstructure of Ferromagnetic Solids (Cambridge University Press, Cambridge, 2003).

${ }^{126}$ R. Skomski, J. P. Liu, and D. J. Sellmyer, Phys. Rev. B 60, 7359 (1999).

${ }^{127}$ S. Chikazumi, Physics of Ferromagnetism (Clarendon Press, Oxford, 1997), p. 120.

${ }^{128}$ Note that for Fe single crystals the change of the spontaneous magnetization between 345 and $693 \mathrm{~K}$ is of the order of $10 \%$ (Ref. 127). 